\title{
SOME CHARACTERIZATIONS OF HARMONIC CONVEX FUNCTIONS
}

\author{
MUHAMMAD ASLAM NOOR*, KHALIDA INAYAT NOOR, SABAH IFTIKHAR
}

ABstract. In this paper, we show that the harmonic convex functions have some nice properties, which convex functions enjoy. We also discuss some basic properties of harmonic convex functions. The techniques and ideas of this paper may be a starting point for future research.

\section{INTRODUCTION}

Convexity theory played an important and fundamental role in the developments of various branches of engineering, financial mathematics, economics and optimization. In recent years, the concept of convex functions and its variant forms have been extended and generalized using innovative techniques to study complicated problems. It is well known that convexity is closely related to inequality theory. The optimality conditions of differentiable convex functions are characterized by variational inequalities, the origin of which can be traced back to Euler, Lagrange and Newton. On the other hand, convex functions are related to integral inequalities. which are called the Harmite-Hadamard type integral inequalities. For recent developments, see $[1,3,9,16]$ and reference therein.

Related to the arithmetic means, we have harmonic means. The harmonic means have applications in electrical circuit theory and other branches of sciences. For example, the total resistance of a set of parallel resistors is obtained by adding up the reciprocal of the individual resistance value and then considering the reciprocal of their total. Also the harmonic means are used in developing parallel algorithms for solving various problems, see Noor [5]. A significant class of convex functions, called harmonic convex was introduced by Anderson et al. [1] and Iscan [3], independently. Noor and Noor $[6,7]$ have shown that the optimality conditions of the differentiable harmonic convex functions on the harmonic convex set can be expressed by a class of variational inequalities, which is called the harmonic variational inequality. For recent developments and applications, see [5-7,9-14].

To the best of our knowledge, this field is new one and has not been developed as yet. In this paper, we show that the harmonic convex functions have some nice properties [2], which convex functions enjoy. We have investigated several basic properties of harmonic convex functions. We obtained the necessary and sufficient characterization of a differentiable harmonic convex and harmonic quasi convex functions. It is worth mentioning that the harmonic variational inequalities is a new class of variational inequalities and is not studied much. The interested readers are encouraged to study these harmonic variational inequalities. It is high time to find the applications of these inequalities along with efficient numerical methods.

\section{Preliminaries}

First of all, we recall the following basic concepts.

Definition 2.1. [1]. A set $K \subset \mathbb{R}^{n} \backslash\{0\}$ is said to be a harmonic convex set, if

$$
\frac{x y}{t x+(1-t) y} \in K, \quad \forall x, y \in K, t \in[0,1] .
$$

Received $4^{\text {th }}$ August, 2017; accepted $10^{\text {th }}$ October, 2017; published $1^{\text {st }}$ November, 2017.

2010 Mathematics Subject Classification. 26D15; 26D10; 90C23.

Key words and phrases. Convex functions; general preinvex functions; differentiability; Hermite-Hadamard inequality.

(C) 2017 Authors retain the copyrights of their papers, and all open access articles are distributed under the terms of the Creative Commons Attribution License. 
Definition 2.2. [3]. A function $f: K \rightarrow \mathbb{R}$, where $K$ is a nonempty harmonic convex set in $\mathbb{R}^{n} \backslash\{0\}$. The function $f$ is said to be a harmonic convex function on $K$, if and only if,

$$
f\left(\frac{x y}{t x+(1-t) y}\right) \leq(1-t) f(x)+t f(y), \quad \forall x, y \in K, t \in[0,1] .
$$

The function $f$ is called strictly harmonic convex on $K$, if the above inequality is true as a strict inequality for each distinct $x$ and $y \in K$ and for each $t \in(0,1)$. The function $f: K \rightarrow \mathbb{R}$ is called harmonic concave (strictly harmonic concave) on $K$ if $-f$ is harmonic convex (strictly harmonic convex) on $K$.

\section{Remark 2.1. Geometric Interpretation}

We now consider the geometric interpretation of harmonic convex function. Let $x$ and $y$ be two distinct points in the domain of $f$, and consider the point $\frac{x y}{t x+(1-t) y}$, with $t \in(0,1)$. Note that $(1-t) f(x)+t f(y)$ gives the weighted arithmetic mean of $f(x)$ and $f(y)$, while $f\left(\frac{x y}{t x+(1-t) y}\right)$ gives the value of $f$ at the point $\frac{x y}{t x+(1-t) y}$. So, for a harmonic convex function $f$, the value of $f$ at the points on the path $\frac{x y}{t x+(1-t) y}$ whose initial point is $x$ and terminal point is $y$, is less than or equal to the chord joining the points $(x, f(x))$ and $(y, f(y))$. For a harmonic concave function, the chord is (on or) below the function itself.

Definition 2.3. [16]. A function $f: K \rightarrow \mathbb{R}$, where $K$ is a nonempty harmonic convex set in $\mathbb{R}^{n} \backslash\{0\}$. The function $f$ is said to be a harmonic quasi convex function on $K$, if and only if,

$$
f\left(\frac{x y}{t x+(1-t) y}\right) \leq \max \{f(x), f(y)\}, \quad \forall x, y \in K, \quad t \in[0,1] .
$$

The function $f$ is said to be harmonic quasi concave, if $-f$ is harmonic quasi convex. A function $f$ is harmonic quasi convex, if whenever $f(y) \geq f(x), f(y)$ is greater than or equal to all the values of $f$ at the points of the path $\frac{x y}{t x+(1-t) y}$. A function is said to be strictly harmonic quasi convex, if strict inequality holds for $f(x) \neq f(y)$.

Definition 2.4. [9]. A function $f: K \rightarrow \mathbb{R}$, where $K$ is a harmonic convex set in $\mathbb{R}^{n} \backslash\{0\}$. The function $f$ is said to be a harmonic log-convex function on $K$, if and only if,

$$
f\left(\frac{x y}{t x+(1-t) y}\right) \leq[f(x)]^{1-t}[f(y)]^{t}, \quad \forall x, y \in K, t \in[0,1] .
$$

From (2.1), it follows that

$$
\begin{aligned}
f\left(\frac{x y}{t x+(1-t) y}\right) & \leq[f(x)]^{1-t}[f(y)]^{t} \\
& \leq(1-t) f(x)+t f(y) \\
& \leq \max \{f(x), f(y)\}, \quad \forall x, y \in K, t \in[0,1],
\end{aligned}
$$

This shows that, every harmonic log-convex function is harmonic convex and every harmonic convex function is harmonic quasi convex, but the converse is not true.

Also from (2.1), we have

$$
\log f\left(\frac{x y}{t x+(1-t) y} \leq(1-t) \log f(x)+t \log f(y), \quad \forall x, y \in K, \quad t \in[0,1] .\right.
$$

For the properties and inequalities of harmonic log-convex functions, see [9, 13, 14].

\section{Main Results}

In this section, we discuss some properties of harmonic convex function and harmonic quasi convex function.

Theorem 3.1. If $K_{i}$ is a family of harmonic convex set, then $\cap_{i \in I} K$ is a harmonic convex set. 
Proof. Let $x, y \in \cap_{i \in I} K_{i}, t \in[0,1]$. Then, for each $i \in I, x, y \in K_{i}$, since $K_{i}$ is a harmonic convex set, it follows that

$$
\frac{x y}{t x+(1-t) y} \in K_{i}, \quad \forall i \in I .
$$

Thus

$$
\frac{x y}{t x+(1-t) y} \in \cap_{i \in I} K_{i} .
$$

Hence, $\cap_{i \in I} K_{i}$ is harmonic convex set.

Theorem 3.2. Let $K$ be a harmonic set. If $f_{i}: K \rightarrow \mathbb{R},(i=1,2,3, \ldots, m)$ are harmonic convex functions. Then the function

$$
f=\sum_{i=1}^{m} a_{i} f_{i}, \quad a_{i} \geq 0, \quad i=1,2,3, \ldots, m
$$

is harmonic convex function.

Proof. Let $K$ be a harmonic set. Then $\forall x, y \in K$ and $t \in[0,1]$, we have

$$
\begin{aligned}
f\left(\frac{x y}{t x+(1-t) y}\right) & =\sum_{i=1}^{m} a_{i} f_{i}\left(\frac{x y}{t x+(1-t) y}\right) \\
& \leq \sum_{i=1}^{m} a_{i}\left[(1-t) f_{i}(x)+t f_{i}(y)\right] \\
& =(1-t) \sum_{i=1}^{m} a_{i} f_{i}(x)+t \sum_{i=1}^{m} a_{i} f_{i}(y) \\
& =(1-t) f(x)+t f(y) .
\end{aligned}
$$

This shows that $f$ is a harmonic convex function.

Theorem 3.3. Let $K$ be a harmonic set. If the functions $f_{i}: K \rightarrow \mathbb{R}$ are harmonic convex functions, then $f=\max \left\{f_{i}, i=1,2,3, \ldots, m\right\}$ is also harmonic convex.

Proof. Consider,

$$
\begin{aligned}
f\left(\frac{x y}{t x+(1-t) y}\right) & =\max \left\{f_{i}\left(\frac{x y}{t x+(1-t) y}\right), i=1,2,3, \ldots, m\right\} \\
& =f_{w}\left(\frac{x y}{t x+(1-t) y}\right) \\
& \leq(1-t) f_{w}(x)+t f_{w}(y) \\
& =(1-t) \max \left\{f_{i}(x)\right\}+t \max \left\{f_{i}(y)\right\} \\
& =(1-t) f(x)+t f(y) .
\end{aligned}
$$

This implies that $f(x)$ is harmonic convex function.

Theorem 3.4. Let $K$ be a harmonic set. If the function $f: K \rightarrow \mathbb{R}$ is harmonic convex function and $g: \mathbb{R} \rightarrow \mathbb{R}$ is a linear function, then $f \circ g$ is a harmonic convex function.

Proof. Let $f$ be a harmonic convex function and $g$ be a linear function. Then

$$
\begin{aligned}
(g \circ f)\left(\frac{x y}{t x+(1-t) y}\right) & =g\left(f\left(\frac{x y}{t x+(1-t) y}\right)\right) \\
& \leq g((1-t) f(x)+t f(y) \\
& =(1-t) g(f(x))+t g(f(y)) \\
& =(1-t)(g \circ f)(x)+t(g \circ f)(y) .
\end{aligned}
$$

This shows that $g \circ f$ is a harmonic convex function. 
Theorem 3.5. Let $K$ be a harmonic set. If the function $f: K \rightarrow \mathbb{R}$ is a harmonic quasi convex function and $g: \mathbb{R} \rightarrow \mathbb{R}$ is a nondecreasing function, then $f \circ g$ is a harmonic quasi convex function.

Proof. Let $f$ be a harmonic quasi convex function and $g$ be a nondecreasing function. Then

$$
\begin{aligned}
(g \circ f)\left(\frac{x y}{t x+(1-t) y}\right) & \leq g[\max \{f(x), f(y)\}] \\
& =\max \{g \circ f(x), g \circ f(y)\},
\end{aligned}
$$

which implies that $(g \circ f)$ is a harmonic quasi convex function.

Theorem 3.6. Let $f: K \rightarrow \mathbb{R}$ be a harmonic convex function. If $\mu=\inf _{x \in K} f(x)$, then the set $E=\{x \in K: f(x)=\mu\}$ is a harmonic convex set. If $f$ is strictly harmonic convex function, then $E$ is a singleton.

Proof. Let $x, y \in E$. Since $f$ is harmonic convex function, so

$$
f\left(\frac{x y}{t x+(1-t) y}\right) \leq(1-t) f(x)+t f(y)=\mu,
$$

which implies that $\frac{x y}{t x+(1-t) y} \in E$ and hence $E$ is a harmonic convex set.

For the second part, assume that $f(x)=f(y)=\mu$. Since $K$ is a harmonic convex set, so for $t \in(0,1)$, $\frac{x y}{t x+(1-t) y} \in K$. Further, since $f$ is strictly harmonic convex function, so

$$
f\left(\frac{x y}{t x+(1-t) y}\right)<(1-t) f(x)+t f(y)=\mu .
$$

This contradicts that $\mu=\inf _{x \in K} f(x)$ and hence $E$ is singleton.

Lemma 3.1. Let $K$ be a nonempty harmonic convex set in $\mathbb{R}^{n} \backslash\{0\}$ and let $f: K \rightarrow \mathbb{R}$ be a harmonic convex function. Then the level set

$$
\alpha=\{x \in K: f(x) \leq \alpha, \alpha \in \mathbb{R}\},
$$

is a harmonic convex se.

Proof. Let $x, y \in K_{\alpha}$. Then $f(x) \leq \alpha, \quad f(y) \leq \alpha$.

Consider,

$$
\begin{aligned}
f\left(\frac{x y}{t x+(1-t) y}\right) & \leq(1-t) f(x)+t f(y) \\
& \leq(1-t) \alpha+t \alpha=\alpha .
\end{aligned}
$$

Hence $\frac{x y}{t x+(1-t) y} \in K_{\alpha}$ and therefore $K_{\alpha}$ is a harmonic convex set.

Remark 3.1. We would like to mention that the converse of above result is not true.

Definition 3.1. [2]. Let $K$ be a nonempty in $\mathbb{R}^{n}$ and let $f: K \rightarrow \mathbb{R}$ be a function. Then epigraph of $f$, denoted by $E(f)$, is defined by

$$
E(f)=\{(x, \alpha): x \in K, \alpha \in \mathbb{R}, f(x) \leq \alpha\} .
$$

Theorem 3.7. Let $K$ be a nonempty harmonic convex set in $\mathbb{R}^{n} \backslash\{0\}$ and let $f: K \rightarrow \mathbb{R}$. Then $f$ is harmonic convex, if and only if, $E(f)$ is a harmonic convex set.

Proof. Assume that $f$ is harmonic convex function and let $(x, \alpha),(y, \beta) \in E(f)$. Then $f(x) \leq \alpha$ and $f(y) \leq \beta$.

Consider, for $t \in[0,1]$,

$$
\begin{aligned}
f\left(\frac{x y}{t x+(1-t) y}\right) & \leq(1-t) f(x)+t f(y) \\
& \leq(1-t) \alpha+t \beta
\end{aligned}
$$


Thus $\left(\frac{x y}{t x+(1-t) y},(1-t) \alpha+t \beta\right) \in E(f)$ and hence $E(f)$ is a harmonic convex set. Conversely, assume that $E(f)$ is a harmonic convex set and let $x, y \in K$. Then $(x, f(x)),(y, f(y)) \in E(f)$ and by the harmonic convexity of $E(f)$, we have

$$
\left(\frac{x y}{t x+(1-t) y},(1-t) f(x)+t f(y)\right) \in E(f) .
$$

Thus, $f\left(\frac{x y}{t x+(1-t) y}\right) \leq(1-t) f(x)+t f(y)$ for each $t \in(0,1)$, that is, $f$ is harmonic convex function.

Remark 3.2. If the function $f: K \rightarrow \mathbb{R}$ is harmonic log-convex function on the harmonic convex set $K$, then $E(f)$ is a harmonic convex set.

Theorem 3.8. Let $K$ be a harmonic convex set and let $f: K \rightarrow \mathbb{R}$ be a harmonic convex function. Then any local minimum of $f$ is a global minimum.

Proof. Let $x \in K$ be a local minimum of the harmonic convex function $f$.. Assume the contrary, that is, $f(y)<f(x)$, for some $y \in K$. Since $f$ is harmonic convex function, we have

$$
f\left(\frac{x y}{t x+(1-t) y}\right) \leq(1-t) f(x)+t f(y) \quad \forall x, y \in K, t \in[0,1] .
$$

Thus

$$
f\left(\frac{x y}{t x+(1-t) y}\right)-f(x) \leq t[f(y)-f(x)],
$$

from which it follows that for some small $t>0$,

$$
f\left(\frac{x y}{t x+(1-t) y}\right)<f(x) .
$$

Contradicting the local minimum. Hence every local minimum of $f$ is a global minimum.

Strictly harmonic quasi convex and strictly harmonic quasi concave functions are especially important in nonlinear programming because they ensure that a local minimum and a local maximum over a harmonic convex set is a global minimum and a global maximum, respectively.

Theorem 3.9. Let $K$ be a harmonic convex set and let $f: K \rightarrow \mathbb{R}$ be a strictly harmonic quasi convex function. Consider the problem of minimum of $f(x)$ subject to $x \in K$. If $\bar{x}$ is a local optimal solution, then $\bar{x}$ is also a global optimal solution.

Proof. Assume, on the contrary, that there exists an $\hat{x} \in K$ with $f(\hat{x})<f(\bar{x})$. By the harmonic convexity of $K, \frac{\bar{x} \hat{x}}{t \bar{x}+(1-t) \hat{x}} \in K$ for each $t \in(0,1)$.

Since $\bar{x}$ is a local minimum by assumption,

$$
f(\bar{x})<f\left(\frac{\bar{x} \hat{x}}{t \bar{x}+(1-t) \hat{x}}\right) \quad \forall t \in(0,1) .
$$

But because $f$ is strictly harmonic quasi convex and $f(\hat{x})<f(\bar{x})$, we have

$$
f\left(\frac{\bar{x} \hat{x}}{t \bar{x}+(1-t) \hat{x}}\right)<f(\bar{x}) \quad \forall t \in(0,1) .
$$

This contradicts the local optimality of $\bar{x}$, and the proof is complete.

Lemma 3.2. Let $K$ be a nonempty harmonic convex set in $\mathbb{R}^{n} \backslash\{0\}$. Then a function $f: K \rightarrow \mathbb{R}$ is a harmonic quasi convex function, if and only if, the level set $K_{\alpha}$ defined by (3.1) is a harmonic convex set.

Proof. Suppose that $f$ is harmonic quasi-convex function and let $x, y \in K_{\alpha}$. Therefore $x, y \in K$ and $\max \{f(x), f(y)\} \leq \alpha$. Also $x_{1}=\frac{x y}{t x+(1-t) y} \in K$, since $K$ is a harmonic convex set.

Using the harmonic quasi-convexity of $f$, we have

$$
f\left(\frac{x y}{t x+(1-t) y}\right) \leq \max \{f(x), f(y)\} \leq \alpha .
$$


Hence $x_{1} \in K_{\alpha}$ and therefore $K_{\alpha}$ is harmonic convex set.

Conversely, suppose that $K_{\alpha}$ is harmonic convex set for each real number $\alpha$. Let $x, y \in K_{\alpha}$. Furthermore, let $t \in(0,1)$ and $x_{1}=\frac{x y}{t x+(1-t) y}$. Note that $x, y \in K_{\alpha}$ for $\alpha=\max \{f(x), f(y)\}$. By assumption, $K_{\alpha}$ is harmonic convex set, so that $x_{1} \in K_{\alpha}$. Therefore

$$
f\left(\frac{x y}{t x+(1-t) y}\right) \leq \alpha=\max \{f(x), f(y)\} .
$$

Hence, $f$ is harmonic quasi-convex and the proof is complete.

Remark 3.3. The level set defined as $K_{\alpha}=\{x \in K: f(x) \leq \alpha, \alpha \in \mathbb{R}\}$, is sometimes referred to as a lower-level set, to differentiate it from the upper-level set defined as $K_{\alpha}=\{x \in K: f(x) \geq \alpha, \alpha \in \mathbb{R}\}$, which is harmonic convex set, if and only if, $f$ is harmonic quasi concave.

Theorem 3.10. If the function $f: K \rightarrow \mathbb{R}$ is harmonic convex function such that $f(x)<f(y)$, for all $x, y \in K$, then $f$ is strictly harmonic quasi convex function.

Proof. By the harmonic convexity of $f$, we have

$$
\begin{aligned}
f\left(\frac{x y}{t x+(1-t) y}\right) & \leq(1-t) f(x)+t f(y) \\
& <f(x) ; \quad \text { since } f(x)<f(y),
\end{aligned}
$$

which shows that the harmonic convex function is strictly harmonic quasi convex function.

Definition 3.2. The function $f: K \rightarrow \mathbb{R}$ is said to be harmonic pseudo convex function with respect to a strictly positive function $b(\cdot, \cdot)$ such that

$$
f(y)<f(x) \Rightarrow f\left(\frac{x y}{t x+(1-t) y}\right)<f(x)+t(t-1) b(x, y), \quad \forall x, y \in K, t \in(0,1) .
$$

Theorem 3.11. If the function $f: K \rightarrow \mathbb{R}$ is harmonic convex function such that $f(y)<f(x)$, then $f$ is harmonic pseudo convex function $f$ with respect to a strictly positive function $b(\cdot, \cdot)$.

Proof. Let $f(y)<f(x)$ and let $f$ be a harmonic convex function. Then

$$
\begin{aligned}
f\left(\frac{x y}{t x+(1-t) y}\right) & \leq f(x)+t(f(y)-f(x)) \\
& <f(x)+t(1-t)(f(y)-f(x)) \\
& =f(x)+t(t-1)(f(x)-f(y)) \\
& <f(x)+t(t-1) b(y, x),
\end{aligned}
$$

where $b(x, y)=f(x)-f(y)>0$, the result result.

Remark 3.4. If the function $f: K \rightarrow \mathbb{R}$ is harmonic log-convex function such that $f(y)<f(x)$, then the harmonic log-convex function is harmonic pseudo convex under the same sense.

Theorem 3.12. Let $K$ be a nonempty harmonic convex set in $\mathbb{R}^{n} \backslash\{0\}$ and let $f: K \rightarrow \mathbb{R}$ be strictly harmonic quasi convex function and lower semicontinuous. Then $f$ is harmonic quasi convex function.

Proof. Let $x, y \in K$. If $f(x) \neq f(y)$, then by the strict harmonic quasi convexity of $f$, we must have

$$
f\left(\frac{x y}{t x+(1-t) y}\right)<\max \{f(x), f(y)\} \quad \forall t \in(0,1) .
$$

Now, suppose that $f(x)=f(y)$. To show that $f$ is harmonic quasi convex, we need to show that

$$
f\left(\frac{x y}{t x+(1-t) y}\right) \leq f(y) \quad \forall t \in(0,1) .
$$

By contradiction, suppose that

$$
f\left(\frac{x y}{t x+(1-t) y}\right)>f(y) \quad \text { for some } t \in(0,1) .
$$


Denote $\frac{x y}{t x+(1-t) y}$ by $\bar{x}$. Since $f$ is lower semicontinuous, there exists a $t \in(0,1)$ such that

$$
f(\bar{x})>f\left(\frac{\bar{x} y}{t \bar{x}+(1-t) y}\right)>f(y)=f(x) .
$$

Hence by the strict harmonic quasi convexity of $f$ and since $f\left(\frac{\bar{x} y}{t \bar{x}+(1-t) y}\right)>f(y)$, we have $f(\bar{x})<$ $f\left(\frac{\bar{x} y}{t \bar{x}+(1-t) y}\right)$, contradicting (3.2). This completes the proof.

The following theorem gives a necessary and sufficient characterization of a differentiable harmonic quasi convex function.

Theorem 3.13. Let $K$ be a nonempty harmonic convex set in $\mathbb{R}^{n} \backslash\{0\}$ and let $f: K \rightarrow \mathbb{R}$ be differentiable on $K$. Then $f$ is harmonic quasi convex, if and only if,

$$
f(x) \leq f(y) \Rightarrow\left\langle f^{\prime}(y), \frac{x y}{y-x}\right\rangle \leq 0, \quad \forall x, y \in K .
$$

Proof. Let $f$ be harmonic quasi convex and let $x, y \in K$ be such that

$$
f(x) \leq f(y) \text {. }
$$

Using the Taylor series, we have

$$
f\left(\frac{x y}{x+t(y-x)}\right)=f(y)+t\left\langle f^{\prime}(y), \frac{x y}{y-x}\right\rangle+t\left\|\frac{x y}{y-x}\right\| \alpha\left[y ; t\left(\frac{x y}{y-x}\right)\right] .
$$

where $\alpha\left[y ; t\left(\frac{x y}{y-x}\right)\right] \rightarrow 0$, as $t \rightarrow 0$.

By the harmonic quasi convexity of $f$, we have $f\left(\frac{x y}{(1-t) x+t y}\right) \leq f(y)$ and hence the above equation implies that

$$
t\left\langle f^{\prime}(y), \frac{x y}{y-x}\right\rangle+t\left\|\frac{x y}{y-x}\right\| \alpha\left[y ; t\left(\frac{x y}{y-x}\right)\right] \leq 0 .
$$

Dividing by $t$ and taking the limit in the above inequality as $t \rightarrow 0$, we have

$$
\left\langle f^{\prime}(y), \frac{x y}{y-x}\right\rangle \leq 0 .
$$

Conversely, suppose that $x, y \in K$ and that $f(x) \leq f(y)$. We need to show that $f\left(\frac{x y}{t x+(1-t) y}\right) \leq f(y)$, for all $x \in K$ and $t \in(0,1)$.

We do this by showing that the set $\mathbb{L}=\left\{x^{\prime}: x^{\prime}=\frac{x y}{t x+(1-t) y}, t \in(0,1), f\left(x^{\prime}\right)>f(y)\right\}$ is empty.

By contradiction, suppose that there exists an $x^{\prime} \in \mathbb{L}$. Therefore $x^{\prime}=\frac{x y}{t x+(1-t) y}$ for some $t \in(0,1)$ and $f\left(x^{\prime}\right)>f(y)$.

Since $f$ is differentiable, it is continuous and there must exits a $\delta \in(0,1)$, such that

$$
f\left(\frac{x^{\prime} y}{(1-\mu) x^{\prime}+\mu y}\right)>f(y) \text { for each } \mu \in(\delta, 1),
$$

and $f\left(x^{\prime}\right)>f\left(\frac{x^{\prime} y}{(1-\delta) x^{\prime}+\delta y}\right)$. By this inequality and the mean value theorem, we must have

$$
0<f\left(x^{\prime}\right)-f\left(\frac{x^{\prime} y}{(1-\delta) x^{\prime}+\delta y}\right)=(1-t)\left\langle f^{\prime}(\hat{x}), \frac{x^{\prime} y}{y-x^{\prime}}\right\rangle,
$$

where $\hat{x}=\frac{x^{\prime} y}{\left(1-\mu^{\prime}\right) x^{\prime}+\mu^{\prime} y}$ for some $\mu^{\prime} \in(\delta, 1)$. From it is clear that $f(\hat{x})>f(y)$. Dividing (3.3) by $(1-t)>0$, it follows that $\left\langle f^{\prime}(\hat{x}), \frac{x^{\prime} y}{y-x^{\prime}}\right\rangle>0$, which in turn implies that

$$
\left\langle f^{\prime}(\hat{x}), \frac{x y}{y-x}\right\rangle>0 .
$$

But on the other hand $f(\hat{x})>f(y) \geq f(x)$, and $\hat{x}$ is harmonic combination of $x$ and $y$. By the assumption of the theorem $\left\langle f^{\prime}(\hat{x}), \frac{\hat{x} x}{\hat{x}-x}\right\rangle \leq 0$, and thus we must have

$$
0 \geq\left\langle f^{\prime}(\hat{x}), \frac{x y}{y-x}\right\rangle .
$$

The above inequality is not compatible with (3.4). Therefore, $\mathbb{L}$ is empty, and the proof is complete. 
The following theorem was derived by Noor and Noor $[6,7]$ and it gives a necessary and sufficient characterization of a differentiable harmonic convex function.

Theorem 3.14. [6]. Let $K$ be a harmonic convex set. If $f: K \rightarrow \mathbb{R}$ is a differentiable harmonic convex function on the harmonic convex set $K$, then

(1) $f(y)-f(x) \geq\left\langle f^{\prime}(x), \frac{x y}{x-y}\right\rangle, \quad \forall x, y \in K$

(2) $\left\langle f^{\prime}(x)-f^{\prime}(y), \frac{x y}{x-y}\right\rangle \leq 0, \quad \forall x, y \in K$,

but the converse is not true.

Theorem 3.15. [6]. Let $f$ be a differentiable harmonic convex function on the harmonic convex set $K$. Then $x \in K$ is a minimum of $f$, if and only if, $x \in K$ satisfies

$$
\left\langle f^{\prime}(x), \frac{x y}{x-y}\right\rangle \geq 0, \quad \forall y \in K .
$$

Remark 3.5. The inequality of the type $\left\langle f^{\prime}(x), \frac{x y}{x-y}\right\rangle \geq 0$ is known as harmonic variational inequality, which was introduced by Noor and Noor $[6,7]$.

Definition 3.3. A function $f: K \rightarrow \mathbb{R}$, where $K$ is a nonempty harmonic set $\mathbb{R}^{n} \backslash\{0\}$. The function $f$ is said to be a harmonic pseudo-convex function, if for each $x, y \in K$ with $\left\langle f^{\prime}(y), \frac{x y}{y-x}\right\rangle \geq 0$, we have $f(x) \geq f(y)$; or equivalently, if $f(x)<f(y)$, then $\left\langle f^{\prime}(y), \frac{x y}{y-x}\right\rangle<0$.

Definition 3.4. A function $f: K \rightarrow \mathbb{R}$, where $K$ is a nonempty harmonic set $\mathbb{R}^{n} \backslash\{0\}$. The function $f$ is said to be a harmonic quasi convex function, if for each $x, y \in K$ with $f(x) \leq f(y)$, we have $\left\langle f^{\prime}(y), \frac{x y}{y-x}\right\rangle \leq 0$; or equivalently, if $\left\langle f^{\prime}(y), \frac{x y}{y-x}\right\rangle>0$, then $f(x)>f(y)$.

Theorem 3.16. Let $K$ be a harmonic convex set and $f: K \rightarrow \mathbb{R}$ a differentiable harmonic convex function on the harmonic convex set $K$. If $f(x) \leq f(y), \forall x, y \in K$, then $f$ is harmonic quasi-convex function. Furthermore, if $f(x)<f(y), \forall x, y \in K$, then $f$ is harmonic pseudo-convex function.

Proof. Let $f$ be differentiable harmonic convex function on the harmonic convex set $K$. Then from Theorem 3.14, we have

$$
\left\langle f^{\prime}(y), \frac{x y}{y-x}\right\rangle \leq f(x)-f(y)
$$

If $f(x) \leq f(y)$, then $\left\langle f^{\prime}(y), \frac{x y}{y-x}\right\rangle \leq 0$. Therefore, from Theorem 3.13, we have $f$ is harmonic quasiconvex function.

Similarly, if $f(x)<f(y)$, we also have $\left\langle f^{\prime}(y), \frac{x y}{y-x}\right\rangle<0$. So, from the Definition 3.3, we have $f$ is harmonic pseudo-convex function.

\section{ACKNOWLEDGEMENTS}

The authors would like to thank Rector, COMSATS Institute of Information Technology, Pakistan, for providing excellent research and academic environments.

\section{REFERENCES}

[1] G. D. Anderson, M. K. Vamanamurthy and M. Vuorinen, Generalized convexity and inequalities, J. Math. Anal. Appl., 335(2007), 1294-1308.

[2] M. S. Bazaraa, D. Hanif, C. M. Shetty, et al. Nonlinear Programming Theory and Algorithms (Second Edition)[M]. The United States of America: John Wiley and Sons, 1993.

[3] I. Iscan, Hermite-Hadamard type inequalities for harmonically convex functions. Hacet. J. Math. Stats., 43(6)(2014), 935-942.

[4] C. P. Niculescu and L. E. Persson, Convex Functions and Their Applications, Springer-Verlag, New York, (2006).

[5] M. A. Noor, Advanced Convex Analysis and Optimization, Lecture Notes, CIIT, (2014-2017).

[6] M. A. Noor and K. I. Noor, Harmonic variational inequalities, Appl. Math. Inf. Sci., 10(5)(2016), 1811-1814.

[7] M. A. Noor and K. I. Noor, Some implicit methods for solving harmonic variational inequalities, Inter. J. Anal. App. 12(1)(2016), 10-14

[8] M. A. Noor and K. I. Noor, Auxiliary principle techinique for variational inequalities, Appl. Math. Inf. Sci., 11(1)(2017), 165-169.

[9] M. A. Noor, K. I. Noor and M. U. Awan. Some characterizations of harmonically log-convex functions. Proc. Jangjeon. Math. Soc., 17(1)(2014), 51-61. 
[10] M. A. Noor, K. I. Noor and S. Iftikhar, Hermite-Hadamard inequalities for harmonic nonconvex functions, MAGNT Research Report. 4(1)(2016), 24-40.

[11] M. A. Noor, K. I. Noor and S. Iftikhar, Integral inequalities for differentiable relative harmonic preinvex functions(survey), TWMS J. Pure Appl. Math. 7(1)(2016),3-19.

[12] M. A. Noor, K. I. Noor and S. Iftikhar, Integral inequalities of Hermite-Hadamard type for harmonic $(h, s)$-convex functions, Int. J. Anal. Appl., 11(1)(2016), 61-69.

[13] M. A. Noor, K. I. Noor, S. Iftikhar and C. Ionescu, Some integral inequalities for product of harmonic log-convex functions, U.P.B.Sci. Bull., Series A,78(4)(2016),11-19.

[14] M. A. Noor, K. I. Noor, S. Iftikhar and C. Ionescu ,Hermite-Hadmard inequalities for co-ordinated harmonic comvex functions, U.P.B.Sci. Bull., Series A,79(1)(2017),24-34.

[15] J. Pecaric, F. Proschan, and Y. L. Tong, Convex Functions, Partial Orderings and Statistical Applications, Acdemic Press, New york, (1992).

[16] T.-Y. Zhang, Ai-P. Ji, F. Qi, Integral inequalities of Hermite-Hadamard type for harmonically quasi-convex functions, Proc. Jangjeon. Math. Soc., 16(3)(2013), 399-407.

${ }^{2}$ Department of Mathematics, COMSAts institute of Information Technology, Islamabad, Pakistan.

${ }^{*}$ Corresponding AUthor: noormaslam@gmail.com 